\title{
LPS Promotes Vascular Smooth Muscle Cells Proliferation Through the TLR4/Rac1/Akt Signalling Pathway
}

\author{
Qianran Yin ${ }^{a}$ Dehua Jiang ${ }^{b}$ Lei Lic Yu Yang ${ }^{b}$ Pei wu ${ }^{a}$ Yuanyuan Luo ${ }^{b}$ \\ Rongli Yang c Dongye Lia,b
}

anstitute of Cardiovascular Disease Research, Xuzhou Medical University, Xuzhou, Jiangsu, bepartment of Cardiology, 'Department of Gerontology, The Affiliated Hospital of Xuzhou Medical University,

Xuzhou, Jiangsu, PR China

\section{Key Words}

Lps - Vascular smooth muscle cells - Proliferation - Phenotypic modulation - Signalling pathways

\begin{abstract}
Background/Aims: Lipopolysaccharide (LPS) is a potent activator of vascular smooth muscle cells (VSMCs) proliferation, but the underlying mechanism remains unknown. In this study, we knocked down Toll-like receptor 4 (TLR4) and Ras-related C3 botulinum toxin substrate 1 (Rac1) expression using small interfering RNA (siRNA) in order to investigate the effects and possible mechanisms of LPS-induced VSMCs proliferation. Methods: VSMCs proliferation was monitored by 5-ethynyl-2'-deoxyuridine staining, and Rac1 activity was measured via Glutathione S-transferase pull-down assay. mRNAs encoding proliferating cell nuclear antigen (PCNA), smooth muscle $22 \alpha(\mathrm{SM} 22 \alpha)$, myosin heavy chain (MYH) and transient receptor potential channel 1 (TRPC1) were detected by qRT-PCR. The expression of total Akt, p-Akt (308), p-Akt (473), SM22 $\alpha$, MYH and TRPC1 protein was analysed by Western blot. Results: Treatment with TLR4 siRNA (siTLR4) or Rac1 siRNA (siRac1) significantly decreased LPS-induced VSMCs proliferation. Moreover, LPS-induced activation of Rac1 through TLR4 was observed. Western blot analysis revealed that transfection with siTLR4 or siRac1 inhibited LPS-induced Akt phosphorylation. We discovered that LPS stimulated VSMCs proliferation via phenotypic modulation and that this effect was partially inhibited by pre-treatment with siTLR4 or siRac1. Further, TLR4 and Rac1 are involved in LPS-induced activation of TRPC1. Conclusion: This study suggests that LPS exerts an effect on VSMCs proliferation and that the TLR4/Rac1/Akt signalling pathway mediates this effect.

\section{Introduction}

The proliferation of vascular smooth muscle cells (VSMCs) plays a pivotal role in the course of various vascular disorders, including vascular remodelling, restenosis and stroke [1]. Inflammation is an important force in the progression of intimal hyperplasia [2]. Toll-

Q. Yin, D. Jiang and L. Li contributed equally to this work.

Dongye Li
and Rongli Yang.

KARGER
Institute of Cardiovascular Disease Research, Xuzhou Medical University, Department of Cardiology, Department of Gerontology, The Affiliated Hospital of Xuzhou Medical University, Xuzhou, Jiangsu, (PR China); E-Mail dongyeli@xzhmu.edu.cn 
like receptor (TLR) 4, an important member of the TLR family, can be activated during these responses [3]. Lipopolysaccharide (LPS) is a widely used exogenous ligand that activates TLR4 and has been extensively studied in immune and inflammatory diseases [4-7]. Recently, much attention has been given to the role of LPS in the initiation and progression of cardiovascular diseases. There is increasing evidence that LPS can induce an inflammatory response by VSMCs via a TLR4-dependent mechanism [8]. Particular attention has been paid to the link between LPS-mediated inflammation and VSMCs proliferation. While LPS has been shown to promote VSMCs proliferation, the mechanism underlying this phenomenon remain poorly investigated [9].

Ras-related C3 botulinum toxin substrate 1 (Rac1) is known to be involved in LPSmediated TLR4 expression [10]. Furthermore, phosphatidylinositol 3-kinase (PI3K) has been shown to mediate TLR4-induced activation of NF-kB in endothelial cells [11]. As a classical intracellular messenger, Akt is involved in VSMCs proliferation associated with inflammatory stimuli such as angiotensin II and platelet derived growth factor. The absence of Akt reduces VSMCs proliferation and migration [12]. In addition, Hattori et al. reported that LPS led to PI3K/Akt pathway activation in VSMCs [13]. However, the relationship among the Rac1, Akt and TLR signalling pathways during LPS-induced VSMCs proliferation has been rarely explored.

Phenotypic modulation of VSMCs from the contractile phenotype (differentiated type) to the synthetic phenotype (dedifferentiated type) is known to be stimulated in different conditions, which is beneficial for VSMCs proliferation [14]. Myosin heavy chain (MYH) is a phenotypic marker of dedifferentiated VSMCs, whereas smooth muscle $22 \alpha$ (SM22 $\alpha$ ) is a marker of differentiated VSMCs. Whether LPS directly stimulates VSMC phenotypic modulation during VSMCs proliferation is unknown.

Our previous study revealed that LPS contributes to VSMCs proliferation via the TLR4/ Rac1/Akt signalling pathway [15]. In the current study, we use small interfering RNA (siRNA) to provide further evidence for the involvement of the TLR4/Rac1/Akt signalling pathway in LPS-mediated VSMCs proliferation. Furthermore, we examine the role of VSMCs phenotypic modulation in VSMCs proliferation following LPS stimulation and explore the role of related molecular mechanisms.

\section{Materials and Methods}

\section{Cell Culture}

VSMCs were isolated from thoracic aortas resected from Sprague-Dawley rats using an explant

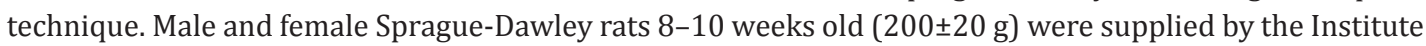
of Laboratory Animals of Xuzhou Medical University. All experimental procedures were authorized by the Animal Ethics Committee of Xuzhou Medical University (CMCACUC2013-04-118), and all methods were carried out consistent with the approved guidelines. VSMCs were used between passages 3 and 5 after culture in Dulbecco's modified Eagle medium (HyClone, USA) supplemented with 10\% heat-inactivated fetal bovine serum (Gibco, USA), $100 \mathrm{U} / \mathrm{ml}$ penicillin, and $100 \mathrm{mg} / \mathrm{ml}$ streptomycin in air supplemented with $5 \% \mathrm{CO}_{2}$ at $37^{\circ} \mathrm{C}$. All cells were synchronized by serum starvation for $24 \mathrm{~h}$.

\section{SiRNA-TLR4 and siRNA-Rac1 Transfection}

VSMCs grown on six-well plates were transfected with either siRNA-TLR4 (siTLR4) or siRac1 from GenePharma (China) according to the manufacturer's instructions. Lipofectamine 2000 (Invitrogen, USA) was used as the transfection reagent. The following siRNA oligos were used in Table 1.

\section{5-ethynyl-2'-deoxyuridine (EdU) Staining}

The Cell-Light TM EdU Kit (Rui Bo Guangzhou Biotechnology Limited Company, China) was used to detect VSMCs proliferation. VSMCs were seeded in six-well plates and stimulated with LPS (10 $\mu \mathrm{g} / \mathrm{ml}$, Sigma, USA; $98 \%$ purity) following siRNA transfection or without transfection as a control. Each well was treated with $500 \mu \mathrm{EdU}(50 \mathrm{mM})$ and incubated for $2 \mathrm{~h}$. EdU-positive cells were examined by fluorescence mi- 


\section{Cellular Physiology Cell Physiol Biochem 2017;44:2189-2200 \begin{tabular}{l|l|l} 
and Biochemistry & Dublished online: Dezember 13, 2017 & $\begin{array}{l}\text { (c) } 2017 \text { The Author(s). Published by S. Karger AG, Basel } \\
\text { www.karger.com/cpb }\end{array}$
\end{tabular}

croscopy (Olympus BX51 microscope, Japan). Images of cells dyed with Apollo 567 were captured with a 'red' filter, while Hoechst 33342-stained cell images were captured with a 'blue' filter. The percentage of EdU-positive cells was calculated using Image-Pro Plus version 6.0 for Windows (Media Cybernetics, Inc, USA) according to the manufacture's protocol as previously described [15].

\section{Quantitative Real-Time Polymerase Chain Reaction ( $q R T-P C R$ ) \\ Total RNA was isolated from VSMCsusing a TRIzol} reagent kit (Invitrogen, USA). cDNA samples were obtained using Script M-MLV (Tiangen Biochemical Technology, China). qRT-PCR was performed using $2 \times$ Super Real PreMix (SYBR Green, Tiangen) and $50 \times$ ROX Reference Dye on an ABI 7500 QPCR System (Applied Biosystems, USA) as previously described [15]. The results were normalized to the expression levels of $\beta$-actin prior to further analysis. Relative gene expression was quantified by the comparative CT method $\left(2^{-\Delta \Delta C T}\right)$. PCR primers for TLR4, Rac1, MYH, SM2 $2 \alpha$, proliferating cell nuclear antigen (PCNA), TRPC1 and $\beta$-actin were designed by Invitrogen with the following sequences in Table 2.

\section{Western Blot Analysis}

The protein levels of Rac1, MYH, SM22 $\alpha$, Akt, p-Akt (308) and p-Akt (473) in VSMCs were analysed by Western blot. Cells were lysed in RIPA Lysis Buffer (Beyotime Institute of Biotechnology, China), and protein was quantified using BCA protein assay reagent (Beyotime). Proteins $(25 \mu \mathrm{g})$ were resolved on $6 \%$ or $10 \%$ SDS-polyacrylamide gels and transferred to polyvinylidene fluoride membranes, which were blocked with $5 \%$ BSA. The primary antibodies antiRac1/2/3(1:1000), anti-p-Akt (308; 1:1000), anti-p-

anti-TRPC1 (1:1000) (all from Santa Cruz, USA) were incubated with polyvinylidene fluoride membranes overnight at $4^{\circ} \mathrm{C}$. After successive washes, membranes were incubated for $1 \mathrm{~h}$ with HRP-conjugated sheep anti-rabbit IgG (1:1000) and horse anti-mouse IgG (1:1000) at room temperature (Zhongshan Golden Bridge Biotechnology Company, China). Enhanced chemiluminescence developing methods (Beyotime) were used to visualize immunolabelling. Image-Pro Plus was used to take measurements of band density.

\section{Pull-Down Assay for Rac1 Activity}

Glutathione S-transferase (p21-activated kinase)-p21 binding domain [GST-(PAK)-PBD] fusion protein was used to measure Rac1 activation in pull-down assays. VSMCs were cultured in six-well plates and stimulated with LPS $(10 \mu \mathrm{g} / \mathrm{ml})$ following siTLR4 transfection or no transfection as a control. VSMCs were lysed, and the supernatant was collected and incubated with GST-(PAK)-PBD fusion protein. After a series of centrifugation and wash steps, the entire complex was eluted from the affinity support using SDS-PAGE loading buffer, and then evaluated by SDS-PAGE. Active Rac1 was detected by Western blotting with mouse anti-Rac1 antibody (New East Biosciences, UK) and HRP-conjugated secondary antibody as previously described [15]. 
Statistical Analysis

All results were expressed as the means \pm standard error of the mean (SEM) of at least three independent experiments. Statistical significance was estimated by one-way or two-way ANOVA with subsequent Newman-Keuls tests for multiple comparisons, while the two-tailed Student's t-test was used to compare data from only two groups. Differences were considered statistically significant at $P<0.05$.

\section{Results}

\section{Transfection of siTLR4 or siRac1 in VSMCS}

Three siRNA options were tested for each gene target. RT-PCR was conducted to determine the efficiency of transfection (Fig. 1). After transfection with siRNA for $48 \mathrm{~h}$, compared with control, the mRNA level of TLR4 or Rac1 decreased individually, indicating successful transfection

Fig. 1. Detection of siTLR4 or siRac1 Transfection in VSMCs. RT-PCR was conducted to detect the efficiency of transfection. Transfection with siTLR4 or siRac1 resulted in satisfactory transfection efficiency, respectively. $\mathrm{n}=3$. * $\mathrm{P}<0.05$ compared with the control group; $* * * \mathrm{P}<0.001 \quad$ compared with the control group.
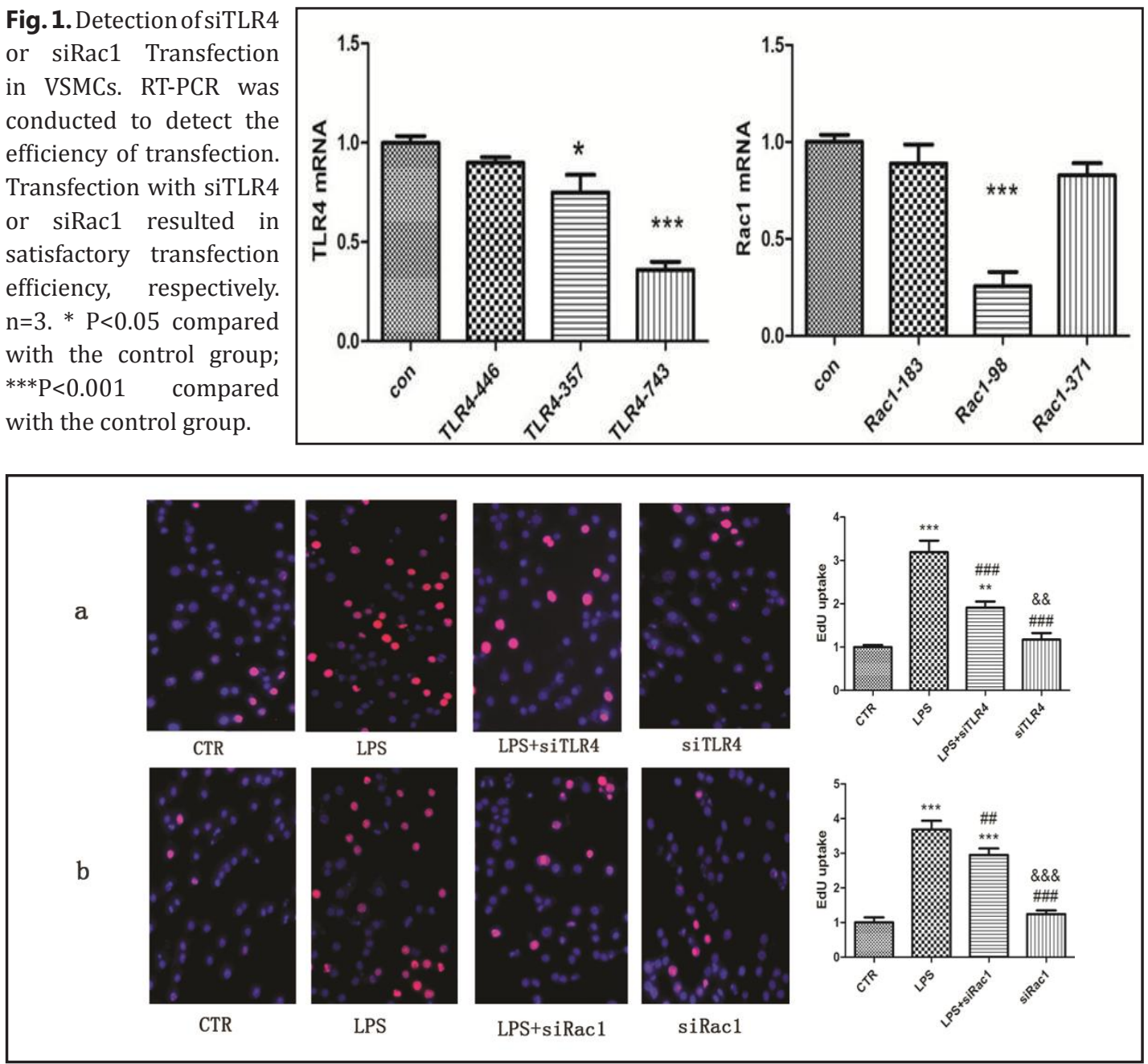

Fig. 2. Detection of incorporated EdU in the DNA of cultured VSMCs. The percentage of EdU-positive cells among LPS-stimulated VSMCs was higher than that obtained without stimulation, and the percentage of EdU-positive cells was reduced after transfection with siTLR4 or siRac1 compared with the LPS-stimulated group. $\mathrm{n}=3$. ${ }^{* *}$ compared with the control group, $\mathrm{P}<0.01,{ }^{* * *} \mathrm{P}<0.001$; \#\# compared with the LPS group $\mathrm{P}<0.01$, \#\#\# $\mathrm{P}<0.001 ;$ \& \& compared with the LPS + siTLR4/siRac1 group, $\mathrm{P}<0.01 ; \& \& \& \mathrm{P}<0.001$.

\section{KARGER}




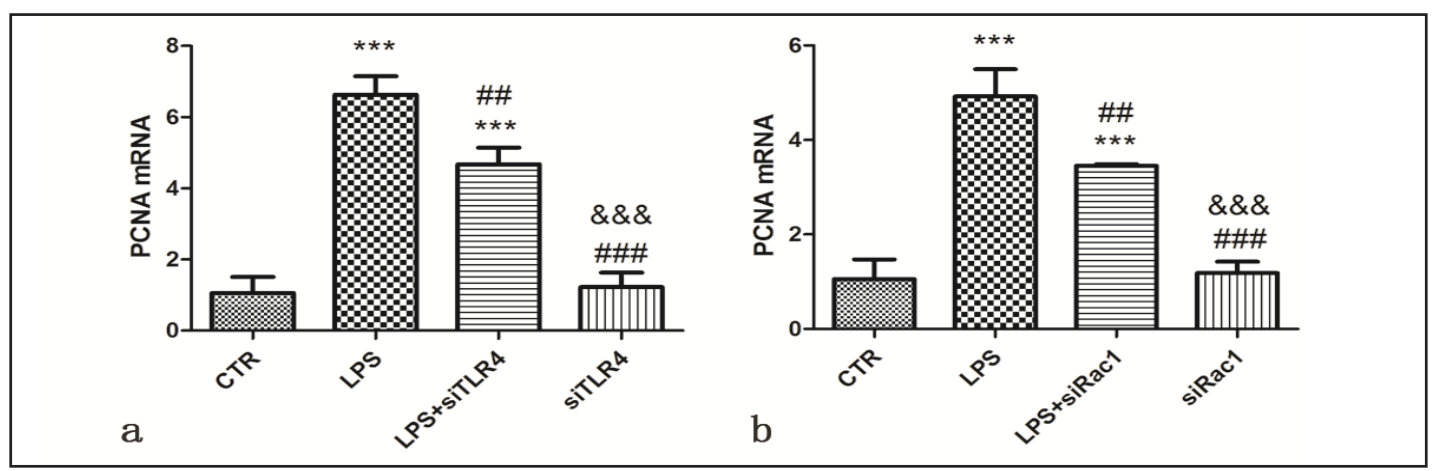

Fig. 3. qRT-PCR for PCNA mRNA expression in VSMCs. We observed high expression of PCNA mRNA after LPS stimulation. Transfection with siTLR4 or siRac1 partially inhibited LPS-mediated expression of PCNA mRNA. $n=3$. ${ }^{* * *} \mathrm{P}<0.001$ compared with the control group; \#\# $\mathrm{P}<0.01$ compared with the LPS group, \#\#\# $\mathrm{P}<0.001 ; \& \& \& \mathrm{P}<0.001$ compared with the LPS + siTLR4/ siRac1 group

Fig. 4. Requirement of TLR4 in Rac1 activation. There was transient activation of Rac1 after stimulation with $10 \mu \mathrm{g} / \mathrm{ml}$ LPS for $15 \mathrm{~min}$. Transfection of VSMCs with siTLR4 reduced LPSmediated GTP-Rac1 expression. $\mathrm{n}=3$. ${ }^{* * *} \mathrm{P}<0.001$ compared with the control group; \#\# $\mathrm{P}<0.01$ compared with the LPS group, \#\#\# $\mathrm{P}<0.001$; $\& \& \& P<0.001$ compared with the LPS + siTLR4/ siRac1 group.

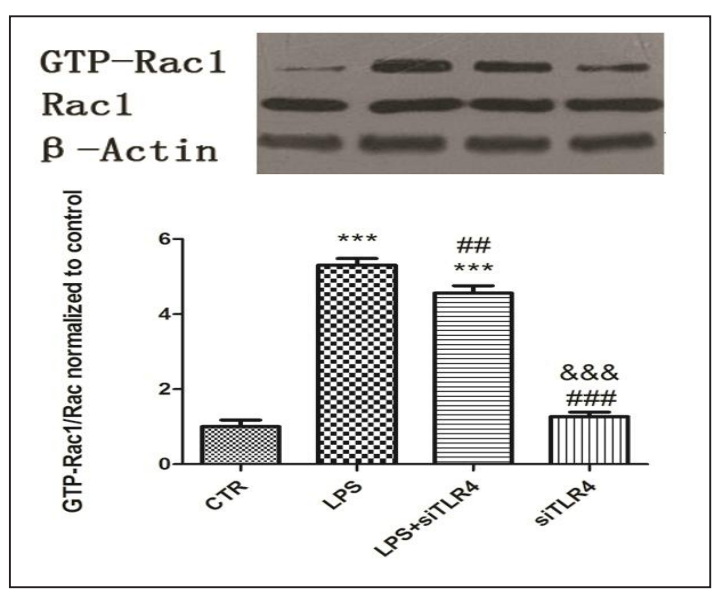

(Fig. 1). siTLR-743 and siRac1-98 were the most effective and were selected for use in subsequent experiments.

\section{Effects of TLR4 and Rac1 on LPS-induced VSMCs proliferation}

EdU assay showed that the number of EdU-positive VSMCs increased significantly after $10 \mu \mathrm{g} / \mathrm{ml}$ LPS treatment, a figure that was reduced after transfection with siTLR4 (Fig. 2a) or siRac1 (Fig. 2b). In parallel, PCNA, a sensitive marker of proliferation in many cell lines, was assayed by qRT-PCR. We found that transfection of siTLR4 (Fig. 3a) or siRac1 (Fig. 3b) partially inhibited LPS-mediated expression of PCNA mRNA. These results suggest that TLR4 and Rac1 are required for LPS-stimulated VSMCs proliferation.

\section{LPS induces the activation of Rac1 through TLR4}

GST pull-down assays were performed to detect Rac1 activation. LPS activated Rac1 for 15 min after stimulation, while transfection with siTLR4 reduced LPS-mediated GTP-Rac1 expression in VSMCs (Fig. 4), suggesting that LPS-induced activation of Rac1 was partially blocked by siTLR4.

\section{Transfection of siTLR4 or siRac1 Inhibits Akt Phosphorylation Stimulated by LPS}

To determine whether TLR4 or Rac1 is required for LPS-mediated Akt activation, the expression of p-Akt was examined by Western blot. Protein expression of p-Akt (308) and p-Akt 


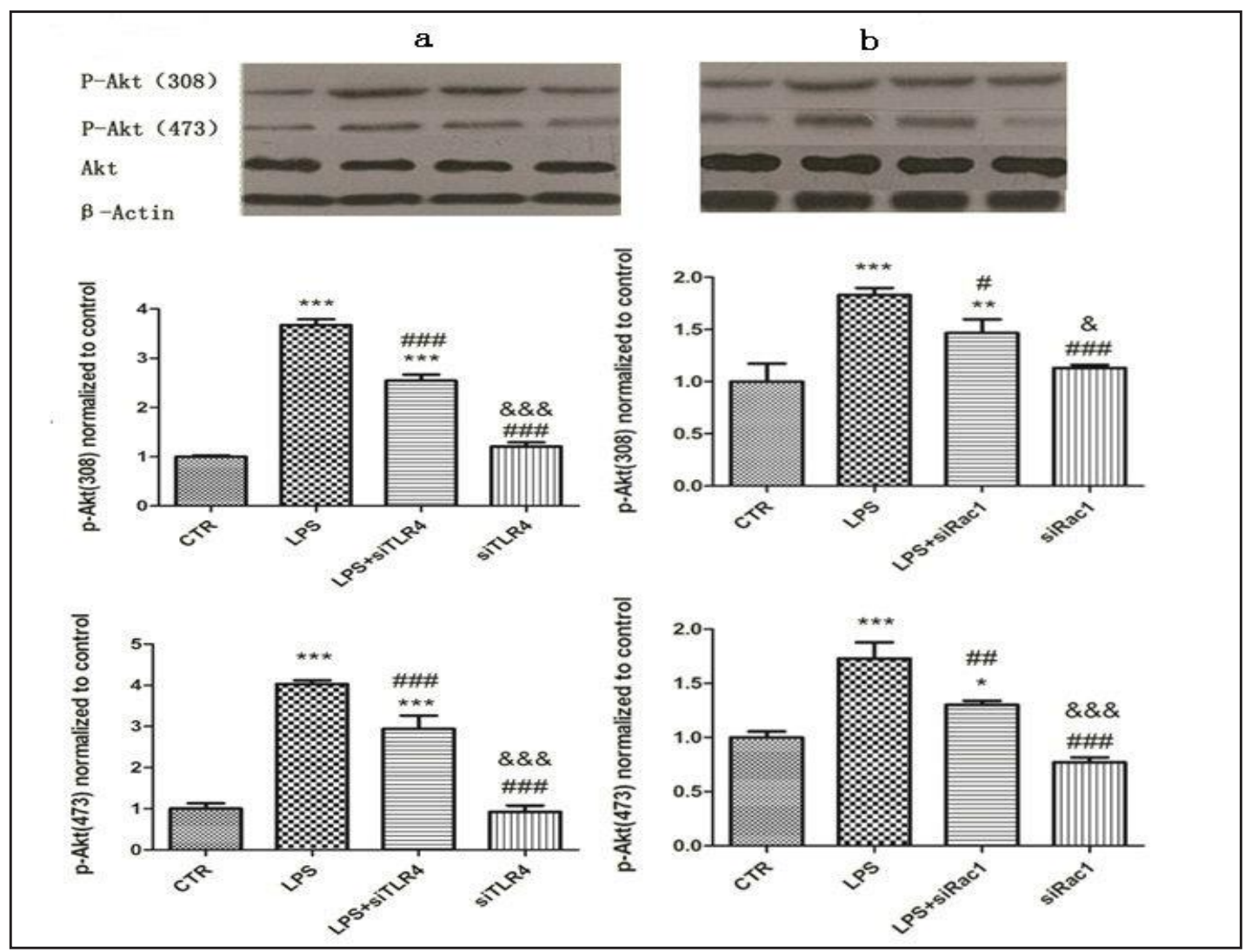

Fig. 5. Inhibition of TLR4 or Rac1 decreases the expression of p-Akt. The expression of p-Akt (308) and p-Akt (473) protein was elevated after stimulation with LPS and diminished significantly after transfection with siTLR4 and siRac1. $\mathrm{n}=3$. $^{*} \mathrm{P}<0.05$ compared with the control group, ${ }^{* * *} \mathrm{P}<0.001 ; \# \mathrm{P}<0.05$ compared with the LPS group, \#\# $\mathrm{P}<0.01$, \#\#\# $\mathrm{P}<0.001$; \& $\mathrm{P}<0.05$ compared with the LPS+siTLR4/siRac1 group; $\& \& \& \mathrm{P}<0.001$.

(473) was elevated after treatment with LPS. Pre-treatment with siTLR4 (Fig. 5a) or siRac1 (Fig. $5 b)$ partially reduced LPS-induced expression of p-Akt (308) and p-Akt (473), indicating that TLR4 and Rac1 mediate the LPS-induced expression of p-Akt (308) and p-Akt (473) in VSMCs.

Phenotypic Modulation Initiates LPS-Induced VSMCs Proliferation through TLR4 and Rac1

LPS reduced mRNA and protein expression of the differentiation marker SM22 $\alpha$, whereas it increased the mRNA and protein levels of the dedifferentiation marker MYH (Figures 6 and 7). However, compared with the LPS group, transfection with siTLR4 resulted in a significant increase in SM22 $\alpha$ expression and a remarkable decrease in the expression of MYH (Figures 6a and 7a). Similar results were also seen after transfection with siRac1 (Figures 6b and 7b). Thus, TLR4 and Rac1 mediate VSMCs proliferation by initiating the process of phenotypic modulation.

\section{LPS Induces the Activation of TRPC1 through the TLR4/Rac1 pathway}

After stimulation with LPS, TRPC1 mRNA and protein were abundantly expressed. However, transfection with siTLR4 or siRac1 reduced LPS-mediated TRPC1 mRNA expression (Fig. 8a) as well as protein expression (Fig. 8b). We therefore suggest that TLR4 and Rac1 participate in TRPC1 expression in LPS-induced VSMCs. 
Fig. 6. The mRNA expression of MYH and SM22 $\alpha$ in VSMCs. SM $22 \alpha$ mRNA expression dropped sharply and MYH mRNA expression increased significantly after stimulation with LPS. Transfection with siTLR4 (a) or siRac1 (b) partially increased the LPSmediated expression of SM22 $\alpha$ mRNA but inhibited that of MYH. $\mathrm{n}=3 .{ }^{*}$ $\mathrm{P}<0.05$ compared with the control group, ${ }^{* * *} \mathrm{P}<0.001$. \# $\mathrm{P}<0.05$ compared with the LPS group, \#\# $\mathrm{P}<0.01$, \#\#\# $\mathrm{P}<0.001 ; \& \& \mathrm{P}<0.01$ compared with the LPS + siTLR4/siRac1 group; $\& \& \& \mathrm{P}<0.001$.

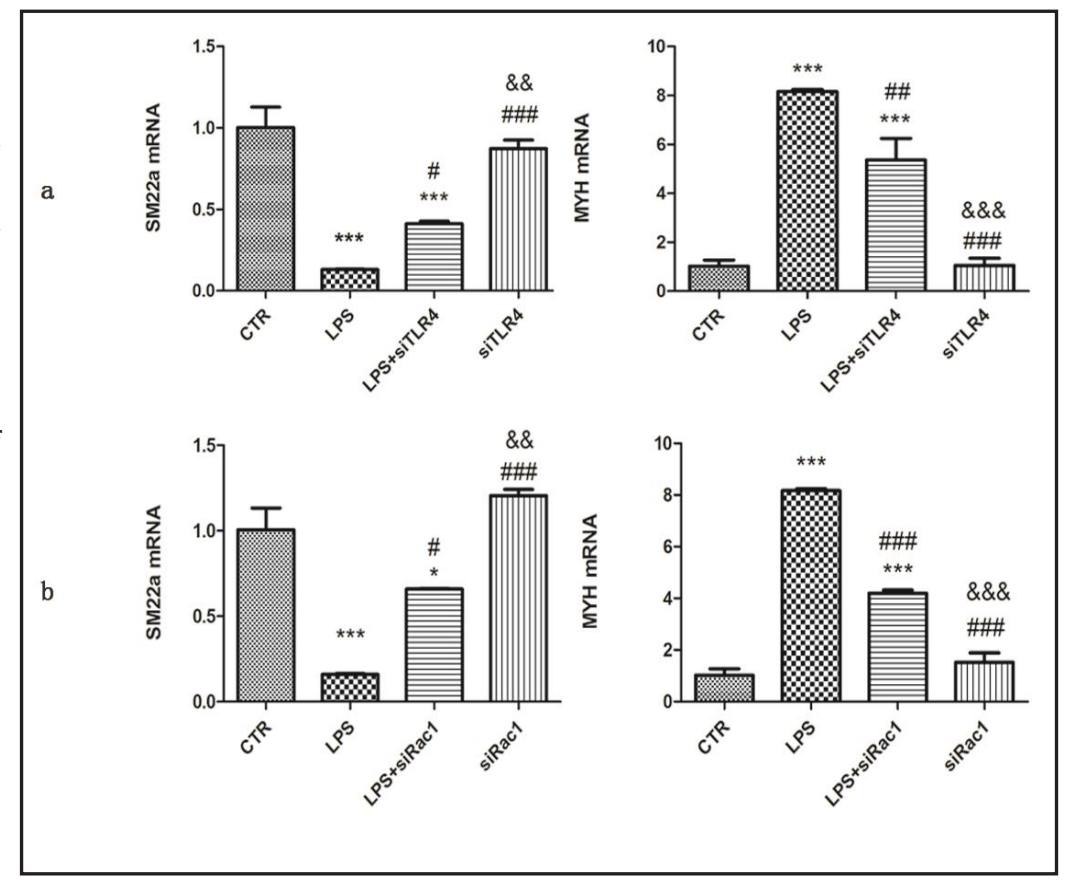

\section{Discussion}

VSMCs proliferation is involved in atherosclerosis $[16,17]$, which is a major pathological factor resulting in myocardial infarction and stroke. LPS, as newly recognized inflammatory substance, has a strong proliferative effect on VSMCs. Substantial evidence indicates that TLR4 initiates signalling downstream of LPS stimulation [18] and may participate in the progression of various vascular proliferative diseases, including atherosclerosis and neointima formation [19-21]. Moreover, blocking the progress of atherosclerosis by inhibiting TLR4-mediated signalling has been verified in different animal models [22-25]. LPS has been shown to activate TLR4 and induce various signalling cascades, including PI3K/Akt [13], mitogen-activated protein kinase $[26,27]$ and interleukin-1 receptor associated kinase (IRAK) $[28,29]$; each of which participates in cell proliferation [30]. However, the underlying molecular mechanisms controlling VSMCs proliferation after LPS stimulation remain to be identified.

Rac1 is a classical cell proliferation factor previously identified in other cell lines [31, 32] and has been studied in the context of cerebro- and cardio-vascular diseases [33]. Lin et al. reported that Rac1 could increase the expression of TLR4 following LPS activation [9]. Furthermore, Rac1 promoted pulmonary artery smooth muscle cell proliferation [34], while Rac1 inhibition reduced VSMCs proliferation stimulated by platelet-derived growth factor [35]. Attenuation of the PI3K/Akt signalling pathway has been shown to limit neointimal formation in rat vascular injury models [36]. Both Rac1 and Akt are considered to lie downstream of the LPS signalling pathway. Based on this information, TLR4, Rac1 and Akt may be involved in the proliferation of VSMCs following LPS stimulation.

Our previous experiments revealed that LPS contributed to VSMCs proliferation via the TLR4/Rac1/Akt signalling pathway [15]. To build on this previous study, we used siRNA to examine the effect of LPS and the role of TLR4 and Rac1 in VSMCs proliferation. LPS was found to promote the proliferation of VSMCs by EdU staining and pre-treatment with siTLR4 or siRac1 significantly decreased LPS-induced VSMCs proliferation. Similarly, LPS-enhanced expression of PCNA mRNA, an indicator of cell proliferation, was diminished after pretreatment with siTLR4 or siRac1. These findings suggest that the expression of TLR4 and 
Rac1 may be involved in the promotion of VSMCs proliferation by LPS. In the present study, siRNA inhibition of TLR4 expression resulted in the down-regulation of Rac1 activity. Thus, TLR4 expression is associated with the regulation of LPS-induced Rac1 activation. Similarly, protein expression of p-Akt (308) and p-Akt (473) was diminished significantly after pretreatment with siTLR4. We found that expression of p-Akt (308) and p-Akt (473) was partly inhibited by transfection with siRac1, consistent with the finding that Rac1 initiated Akt activation during VSMCs proliferation [15]. This indicates that Rac1 activity is associated with the regulation of Akt activation induced by LPS. In conclusion, our results reveal that the effect of LPS on VSMCs proliferation is mediated by the TLR4/Rac1/Akt signalling pathway.

Phenotypic modulation, which means switching from a contractile to a synthetic phenotype, is observed upon vascular injury and in various vascular diseases, including atherosclerosis and hypertension [37]. To investigate whether phenotypic modulation is involved in LPS-induced VSMCs proliferation, Rac1 and TLR4 were knocked down by siRNA. Our results revealed that LPS increases the levels of MYH mRNA and protein while decreasing expression of SM22 $\alpha$ mRNA and protein. When cells were transfected with siTLR4 prior to LPS stimulation, the expression of MYH mRNA and protein were decreased compared with the LPS group, and SM22 $\alpha$ mRNA and protein levels increased. We also knocked down Rac1 with siRNA and examined changes in the mRNA and protein levels of MYH and SM22 $\alpha$. Our results were consistent with those obtained for siTLR4 transfection prior to LPS exposure. These findings demonstrate that down-regulating TLR4 or Rac1 inhibited LPS-mediated VSMCs dedifferentiation. Taken together, these results suggest that phenotypic modulation of VSMCs participates in the proliferation of VSMCs following LPS stimulation.

Fig. 7. TLR4 and Rac1 are required for protein expression of phenotypic modulation markers. Inhibition of TLR4 (a) or Rac1 (b) significantly increased LPSmediated SM22 $\alpha$ protein expression but reduced that of MYH. $n=3 .{ }^{*} \mathrm{P}<0.05$ compared with the control group, ** $\quad \mathrm{P}<0.01, \quad * * *$ $\mathrm{P}<0.001$. \#\# $\mathrm{P}<0.01$ compared with the LPS group, \#\#\# $\mathrm{P}<0.001$; \& \& $\mathrm{P}<0.01$ compared with the LPS + siTLR4/ siRac1 group; $\& \& \& \mathrm{P}<0.001$.

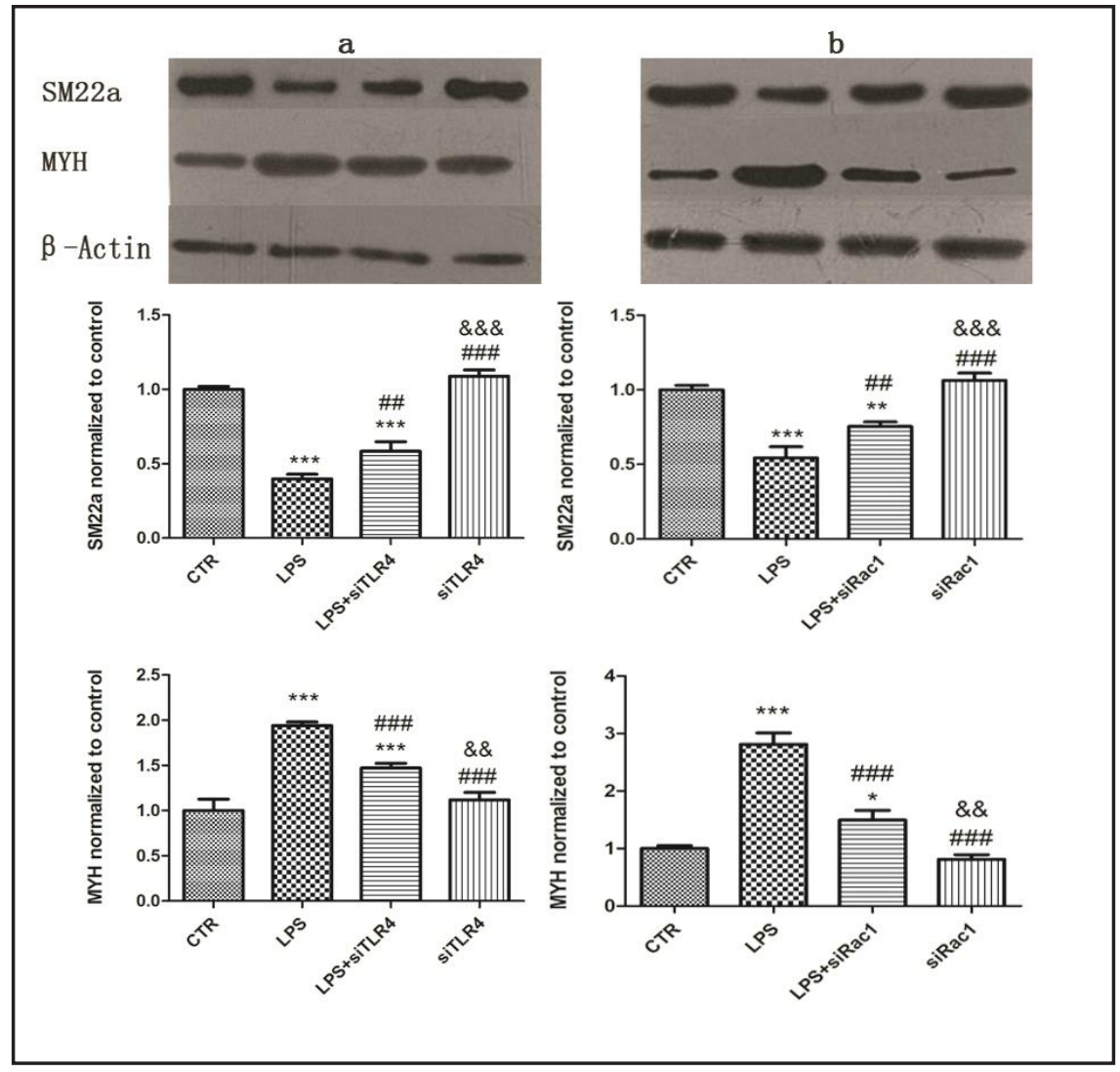


Fig. 8. TLR4 and Rac1 inhibit the expression of TRPC1 induced by LPS. After stimulation with LPS, TRPC1 mRNA was abundantly expressed but dropped dramatically following transfection with siTLR4 or siRac1 (a). Similarly, inhibition of TLR4 or Rac1 mRNA expression reduced LPS-mediated TRPC1 protein expression (b). $n=3$. * $\mathrm{P}<0.1$ compared with the control group; ** $\mathrm{P}<0.01$, *** $\mathrm{P}<0.001$ ; \# $\mathrm{P}<0.1$ compared with the LPS group, \#\# $\quad \mathrm{P}<0.01, \quad$ \#\#\# $\mathrm{P}<0.001 ; \quad$ \& $\quad \mathrm{P}<0.1$ compared with the LPS + siTLR4 / siRac1 group; \&\&\&P<0.001.

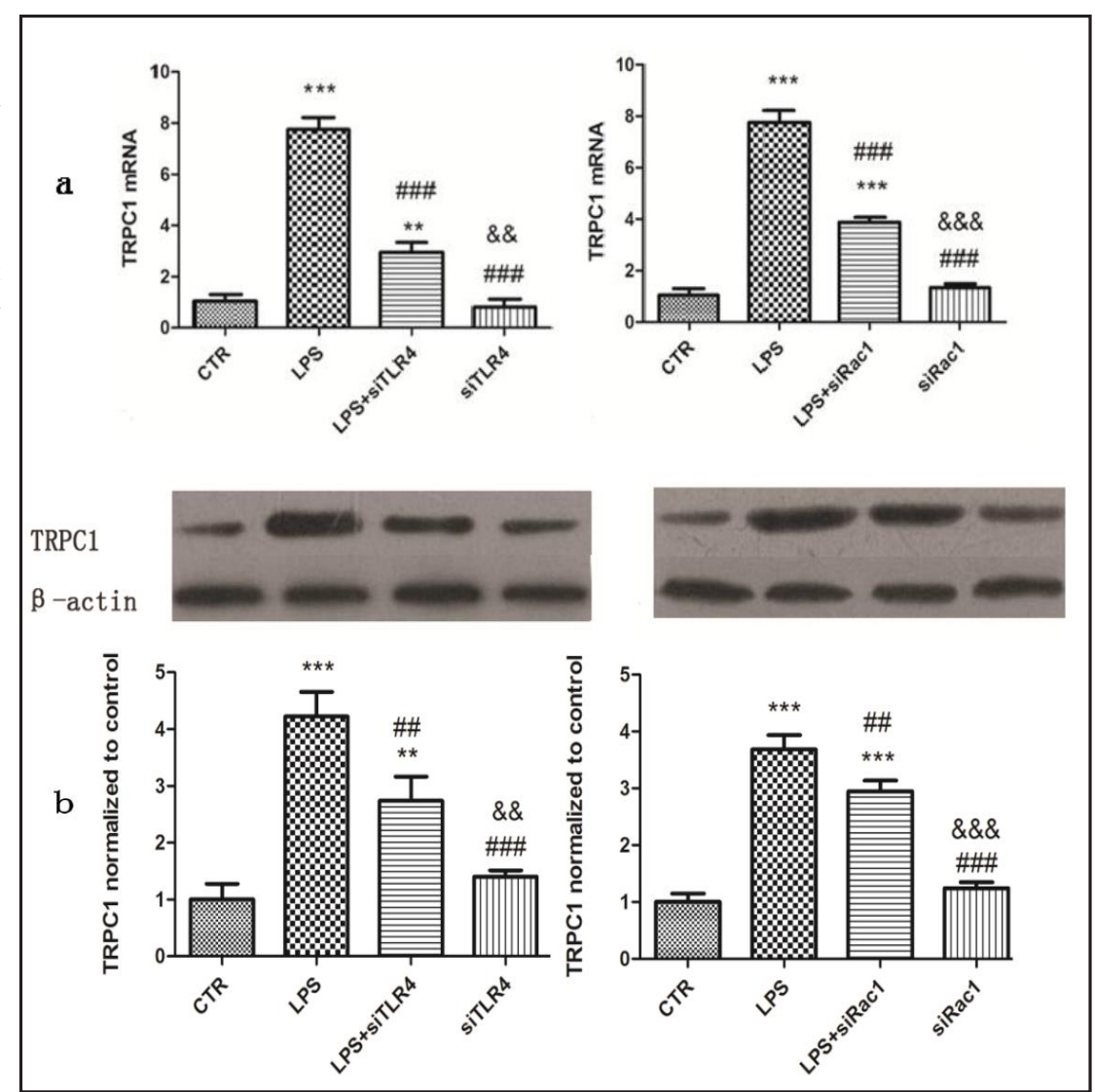

VSMCs express a large repertoire of ion channels that are critical to VSMCs contraction, migration and proliferation. Transient receptor potential canonical (TRPC) 1, a member of the TRPC superfamily, is widely expressed in the VSMCs of several species. TRPC1 has been implicated in mediating several VSMCs pathologies involving contraction and proliferation [38]. Golovina et al. reported that TRPC1 protein expression was increased in proliferative human pulmonary artery smooth muscle cells compared to non-proliferative cells [39]. Takahashi et al. showed that in cultured VSMCs, TRPC1 expression increased upon angiotensin II stimulation $=[40]$. Treatment with antisense oligonucleotides targeting TRPC1 mRNA was able to decrease the proliferation of isolated VSMCs. These findings suggest TRPC1 as a potential target for vascular disease therapies [41]. Jiang et al. have shown that downregulation of TRPC channels reduces LPS-induced pulmonary artery smooth muscle cells proliferation [42]. Kumar et al. have suggested that TRPC1 is up-regulated following vascular injury in mice and pigs in association with VSMCs phenotypic modulation [43]. We hypothesized that TRPC1 participates in phenotypic modulation via the TLR4 and Rac1 signalling pathways, resulting in VSMCs proliferation. In the present study, we discovered that up-regulation of TRPC1 protein and mRNA expression was mediated by LPS. Our experimental results further showed that inhibition of TLR4 or Rac1 by siRNA decreased TRPC1 protein and mRNA expression induced by LPS, suggesting that TLR4 and Rac1 take part in the LPSinduced activation of TRPC1 protein and mRNA. However, how TRPC1 is activated in this process will require more investigation.

In our previous study, we used pharmacological approaches to show how LPS activated TLR4 to trigger VSMCs proliferation, which was involved in Rac1 and Akt cascade [15]. We reported that Rac1 activity is essential for LPS-induced p-Akt expression. However, without a TLR4 inhibitor or siRNA-mediated knock-down of TLR4, the TLR4-independent effects of LPS remain unknown. In the present study, we confirm that LPS significantly promotes 
VSMCs proliferation and that this effect is partially reduced by transfection with siTLR4 or siRac1. The use of small interference technology makes these results more convincing than those obtained via pharmacological approaches. In addition, we verified the requirement for TLR4 in Rac1 activation and formation the TLR4/Rac1/Akt signalling pathway. We did not address whether LPS-induced TLR4 protein and mRNA expressions is mediated by Rac1 and Akt activation, which was emphasized in our previous study. In summary, we provide convincing evidence that the TLR4/Rac1/Akt signalling pathway contributes to VSMCs proliferation. Some studies have reported the impact of LPS on the expression of VSMCs differentiation markers. We found that LPS induced the activation of TRPC1 through the TLR4/Rac1 pathway in VSMCs, suggesting a novel target for therapeutic intervention.

In conclusion, our results show that LPS significantly promotes VSMCs proliferation, an effect that is partially reduced by transfection with siTLR4 or siRac1. We confirm that the TLR4/Rac1/Akt signalling pathway contributes to VSMCs proliferation. LPS stimulates VSMCs proliferation as a result of phenotypic modulation; this effect was also reduced by transfection with siTLR4 or siRac1. Finally, we prove that LPS induces the activation of TRPC1 through the TLR4/Rac1 pathway. We have therefore demonstrated that LPS stimulates the proliferation of VSMCs and that the TLR4/Rac1/Akt signalling pathway is involved in this process.

Our experiments are not without limitations. First, we do not examine whether LPS administration is subject to positive feedback regulation through the TLR4/Rac1/Akt signalling pathway in vivo. Second, the precise mechanisms governing the phenotypic modulation of VSMCs remain scarcely due to the complex and redundant molecules and pathways involved. Thus, pharmacological approaches inhibiting the phenotypic modulation of VSMCs may be difficult to develop. Zhang et al. have summarized existing knowledge of the role and molecular mechanisms involved in controlling VSMCs phenotypic modulation. Moreover, they discuss many molecules, including microRNAs, cytokines and biochemical factors, membrane receptors, ion channels, cytoskeleton and extracellular matrix may become a promising target in VSMCs differentiation-related diseases [44]. Bolı'var et al. recently reported that LPS induces TLR4 activation and prevents cardiac fibroblast-tomyofibroblast differentiation and that decreased $\alpha$-SMA expression played a role in this effect [45]. However, whether LPS-induced phenotypic modulation of VSMCs represents a new therapeutic target for proliferative vascular diseases warrants further investigation. How these strategies will transition from bench to bedside is a topic for future study.

\section{Disclosure Statement}

The authors declare no conflict of interest.

\section{Acknowledgements}

Thanks for the excellent technical support of Yang Liu. Dongye Li, Qianran Yin and Dehua Jiang conceived and designed the experiments, Qianran Yin and Lei Li performed the experiments, YuYang, Pei wu and Yuanyuan Luo analyzed the data, Qianran Yin and Dehua Jiang wrote the paper, Dongye Li and Rongli Yang contributed reagents/materials/analysis tools.

\section{References}

1 Curcio A, Torella D, Indolfi C: Mechanisms of smooth muscle cell proliferation and endothelial regeneration after vascular injury and stenting: approach to therapy. Circ J 2011;75:1287-1296.

-2 Lee MY, San Martin A, Mehta PK, Dikalova AE, Garrido AM, Datla SR, Lyons E, Krause KH, Banfi B, Lambeth JD, Lassegue B, Griendling KK: Mechanisms of vascular smooth muscle NADPH oxidase 1 (Nox1) contribution to injury-induced neointimal formation. Arterioscler Thromb Vasc Biol 2009;29:480-487. 


\section{Cellular Physiology Cell Physiol Biochem 2017;44:2189-2200 \begin{tabular}{l|l|l} 
and Biochemistry & Dublished onlIne: Dezember 13, 2017 & $\begin{array}{l}\text { C } 2017 \text { The Author(s). Published by S. Karger AG, Basel } \\
\text { www.karger.com/cpb }\end{array}$
\end{tabular}}

Yin et al.: TLR4/Rac1/Akt Pathway Involved in VSMCs Proliferation

3 Frantz S, Ertl G, Bauersachs J: Mechanisms of disease: Toll-like receptors in cardiovascular disease. Nat Clin Pract Cardiovasc Med 2007;4:444-454.

4 Jialal I, Kaur H: The Role of Toll-Like Receptors in Diabetes-Induced Inflammation: Implications for Vascular Complications. Curr Diab Rep 2012;12:172-179.

-5 Wang X, Yan S, Xu D, Li J, Xie Y, Hou J, Jiang R, Zhang C, Sun B: Aggravated Liver Injury but Attenuated Inflammation in PTPRO-Deficient Mice Following LPS/D-GaIN Induced Fulminant Hepatitis. Cell Physiol Biochem 2015;37:214-224.

6 Xu D, Yan S, Wang H, Gu B, Sun K, Yang X, Sun B, Wang X: IL-29 Enhances LPS/TLR4-Mediated Inflammation in Rheumatoid Arthritis. Cell Physiol Biochem 2015; 37:27-34.

7 Gou Z, Jiang S, Zheng C, Tian Z, Lin X: Equol Inhibits LPS-Induced Oxidative Stress and Enhances the Immune Response in Chicken HD11 Macrophages. Cell Physiol Biochem 2015;36:611-621.

8 Yang X, Coriolan D, Murthy V, Schultz K, Golenbock DT, Beasley D: Proinflammatory phenotype of vascular smooth muscle cells: role of efficient Toll-like receptor 4 signaling. Am J Physiol Heart Circ Physiol 2005;289:H1069-H1076.

-9 Lin FY, Chen YH, Tasi JS, Chen JW, Yang TL, Wang HJ, Li CY, Chen YL, Lin SJ: Endotoxin induces toll-like receptor 4 expression in vascular smooth muscle cells via NADPH oxidase activation and mitogen-activated protein kinase signaling pathways. Arterioscler Thromb Vasc Biol 2006;26:2630-2637.

10 Sanlioglu S, Williams CM, Samavati L, Butler NS, WangG, McCray, PB, Jr Ritchie, TC, Hunninghake GW, Zandi E, Engelhardt JF: Lipopolysaccharide induces Rac1-dependent reactive oxygen species formation and coordinates tumor necrosis factor-alpha secretion through IKK regulation of NF-kappa B. J Biol Chem 2001;276:30188-30198.

11 Li X, Tupper JC, Bannerman DD, Winn RK, Rhodes CJ, Harlan JM: Phosphoinositide 3 kinase mediates Tolllike receptor 4-induced activation of NF-kappa B in endothelial cells. Infect Immun 2003;71:4414-4420.

12 Fernandez-Hernando C, Jozsef L, Jenkins D, Di Lorenzo A, Sessa WC: Absence of Akt1 reduces vascular smooth muscle cell migration and survival and induces features of plaque vulnerability and cardiac dysfunction during atherosclerosis. Arterioscler Thromb Vasc Biol 2009;29:2033-2040.

13 Hattori Y, Hattori S, Kasai K: Lipopolysaccharide activates Akt in vascular smooth muscle cells resulting in induction of inducible nitric oxide synthase through nuclear factor-kappa B activation. Eur J Pharmacol 2003;481:153-158.

14 Shi N, Chen SY: Mechanisms simultaneously regulate smooth muscle proliferation and differentiation. J Biomed Res 2014;28:40-46.

15 Jiang D, Li D, Cao L, Wang L, Zhu S, Xu T, Wang C, Pan D: Positive feedback regulation of proliferation in vascular smooth muscle cells stimulated by lipopolysaccharide is mediated through the TLR 4/Rac1/Akt pathway. PLoS One 2014;9:e92398.

16 Lim S, Park S: Role of vascular smooth muscle cell in the inflammation of atherosclerosis. BMB Rep 2014;47:1-7.

17 Doran AC, Meller N, McNamara CA: Role of smooth muscle cells in the initiation and early progression of atherosclerosis. Arterioscler Thromb Vasc Biol 2008;28:812-819.

-18 Zhang X, Dong H, Zhang S, Lu S, Sun J, Qian Y: Enhancement of LPS-induced microglial inflammation response via TLR4 under high glucose conditions. Cell Physiol Biochem 2015;35:1571-1581.

19 Heo SK, Yun HJ, Noh EK, Park WH, Park SD: LPS induces inflammatory responses in human aortic vascular smooth muscle cells via Toll-like receptor 4 expression and nitric oxide production. Immunol Lett 2008;120:57-64.

20 He Z, Gao Y, Deng Y, Li W, Chen Y, Xing S, Zhao X, Ding J, Wang X: Lipopolysaccharide induces lung fibroblast proliferation through Toll-like receptor 4 signaling and the phosphoinositide3-kinase-Akt pathway. PLoS One 2012;7:e35926.

21 Pryshchep 0, Ma-Krupa W, Younge BR, Goronzy JJ, Weyand CM: Vessel-specific Toll-like receptor profiles in human medium and large arteries. Circulation 2008;118:1276-1284.

22 Devaraj S, Tobias P, Jialal I: Knockout of toll-like receptor-4 attenuates the pro-inflammatory state of diabetes. Cytokine 2011;55:441-445.

23 Ji Y, Liu J, Wang Z, Liu N: Angiotensin II induces inflammatory response partly via toll-like receptor 4-dependent signaling pathway in vascular smooth muscle cells. Cell Physiol Biochem 2009;23:265-276.

24 Li N, Zhang X, Dong H, Zhang S, Sun J, Qian Y: Lithium Ameliorates LPS-Induced Astrocytes Activation Partly via Inhibition of Toll-Like Receptor 4 Expression. Cell Physiol Biochem 2016;38:714-725.

25 Zhou Y, Zhang T, Wang X, Wei X, Chen Y, Guo L, Zhang J, Wang C: Curcumin Modulates Macrophage Polarization Through the Inhibition of the Toll-Like Receptor 4 Expression and its Signaling Pathways. Cell 


\section{Cellular Physiology Cell Physiol Biochem 2017;44:2189-2200

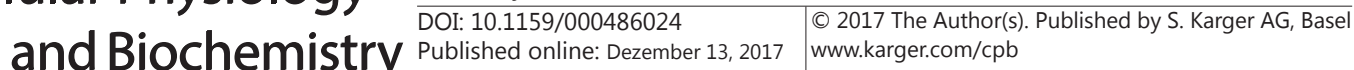

Physiol Biochem 2015;36:631-641.

-26 Son YH, Jeong YT, Lee KA, Choi KH, Kim SM, Rhim BY, Kim K: Roles of MAPK and NF-kappaB in interleukin-6 induction by lipopolysaccharide in vascular smooth muscle cells. J Cardiovasc Pharmacol 2008;51:71-77.

-27 Chen Z, Chen Y, Pan L, Li H, Tu J, Liu C, Dai X, Zhang X, Sun G, Feng D: Dachengqi Decoction Attenuates Inflammatory Response via Inhibiting HMGB1Mediated NF- $\mathrm{BB}$ and P38 MAPK Signaling Pathways in Severe Acute Pancreatitis. Cell Physiol Biochem 2015;37:1379-1389.

-28 Jain M, Singh A, Singh V, Barthwal MK: Involvement of interleukin-1 receptor-associated kinase-1 in vascular smooth muscle cell proliferation and neointimal formation after rat carotid injury. Arterioscler Thromb Vasc Biol 2015;35:1445-1455.

-29 Bai S, Li D, Zhou Z, Cao J, Xu T, Zhang X, Wang Y, Guo J, Zhang Y: Interleukin-1 Receptor-Associated Kinase $1 / 4$ as a Novel Target for Inhibiting Neointimal Formation After Carotid Balloon Injury. J Atheroscler Thromb 2015;22:1317-1337.

-30 Shirasuna K, Shimamura N, Seno K, Ohtsu A, Shiratsuki S, Ohkuchi A, Suzuki H, Matsubara S, Nagayama S, Iwata H, Kuwayama T: Moderate Hypoxia Down-Regulates Interleukin-6 Secretion and TLR4 Expression in Human Sw.71 Placental Cells. Cell Physiol Biochem 2015;36:2149-2160.

-31 Vecchione C, Aretini A, Marino G, Bettarini U, Poulet R, Maffei A, Sbroggio M, Pastore L, Gentile MT, Notte A, Iorio L, Hirsch E, Tarone G, Lembo G: Selective Rac-1 inhibition protects from diabetes-induced vascular injury. Circ Res 2006;98:218-225.

-32 Arbibe L, Mira JP, Teusch N, Kline L, Guha M, Mackman N, Godowski PJ, Ulevitch RJ, Knaus UG: Tolllike receptor 2-mediated NF-kappa B activation requires a Rac1- dependent pathway. Nat Immunol 2000;1:533-540.

33 Carrizzo A, Forte M, Lembo M, Formisano L, Puca AA, Vecchione C:Rac-1 as a new therapeutic target in cerebro- and cardio-vascular diseases. Curr Drug Targets 2014;15:1231-1246.

34 Diebold I, Djordjevic T, Hess J, Gorlach A: Rac-1 promotes pulmonary artery smooth muscle cell proliferation by upregulation of plasminogen activator inhibitor-1: role of NFkappaB-dependent hypoxiainducible factor-1alpha transcription. Thromb Haemost 2008;100:1021-1028.

-35 Kong G, Lee S, Kim KS: Inhibition of rac1 reduces PDGF-induced reactive oxygen species and proliferation in vascular smooth muscle cells. J Korean Med Sci 2001;16:712-718

-36 Miyake H, Maeda K, Asai N, Shibata R, Ichimiya H, Isotani-Sakakibara M, Yamamura Y, Kato K, Enomoto A, Takahashi M, Murohara T: The actin-binding protein Girdin and its Akt-mediated phosphorylation regulate neointima formation after vascular injury. Circ Res 2011;108:1170-1179.

-37 Zhang MJ, Zhou Y, Chen L, Wang YQ, Wang X, Pi Y, Gao CY, Li JC, Zhang LL: An overview of potential molecular mechanisms involved in VSMC phenotypic modulation. Histochem Cell Biol 2016;145:119-130.

-38 Gonzalez-Cobos JC, Trebak M: TRPC channels in smooth muscle cells. Front Biosci 2010;15:1023-1039.

39 Golovina VA, Platoshyn O, Bailey CL, Wang J, Limsuwan A, Sweeney M, Rubin LJ, Yuan JX:Upregulated TRP and enhanced capacitative $\mathrm{Ca}^{(2+)}$ entry in human pulmonary artery myocytes during proliferation. Am J Physiol Heart Circ Physiol 2001;280:H746-755.

40 Takahashi Y, Watanabe H, Murakami M, Ohba T, Radovanovic M, Ono K, Iijima T, Ito H: Involvement of transient receptor potential canonical 1 (TRPC1) in angiotensin II-induced vascular smooth muscle cell hypertrophy. Atherosclerosis 2007;195:287-296.

41 Sweeney M, Yu Y, Platoshyn 0, Zhang S, McDaniel SS, Yuan JX: Inhibition of endogenous TRP1 decreases capacitative $\mathrm{Ca}^{2+}$ entry and attenuates pulmonary artery smooth muscle cell proliferation. Am J Physiol Lung Cell Mol Physiol 2002;283:L144-155.

-42 Jiang HN, Zeng B, Chen GL, Lai B, Lu SH, Qu JM: Lipopolysaccharide potentiates endothelin-1-induced proliferation of pulmonary arterial smooth muscle cells by upregulating TRPC channels. Biomed Pharmacother 2016;82:20-27.

43 Kumar B, Dreja K, Shah SS, Cheong A, Xu SZ, Sukumar P, Naylor J, Forte A, Cipollaro M, McHugh D, Kingston PA, Heagerty AM, Munsch CM, Bergdahl A, Hultgardh-Nilsson A, Gomez MF, Porter KE, Hellstrand P, Beech DJ: Upregulated TRPC1 channel in vascular injury in vivo and its role in human neointimal hyperplasia. Circ Res 2006;98:557-563.

44 Zhang MJ, Zhou Y, Chen L, Wang YQ, Wang X, Pi Y, Gao CY, Li JC, Zhang LL: An overview of potential molecular mechanisms involved in VSMC phenotypic modulation. Histochem Cell Biol 2016;145:119-130.

-45 Bolívar S, Santana R, Ayala P, Landaeta R, Boza P, Humeres C, Vivar R, Muñoz C, Pardo V, Fernandez S, Anfossi R, Diaz-Araya G:Lipopolysaccharide Activates Toll-Like Receptor 4 and Prevents Cardiac Fibroblastto-Myofibroblast Differentiation. Cardiovasc Toxicol 2017. doi: 10.1007/s12012-017-9404-4. 